\title{
Clinical evaluation of enzyme immunoassay in rapid diagnosis of herpes simplex infections
}

\author{
M Sillis
}

\begin{abstract}
Aims: To evaluate the performance of antigen detection by IDEIA (NovoNordisk Ltd) in the rapid diagnosis of potentially serious herpes simplex (HSV) infections. Methods: Nine hundred and twelve specimens from a variety of clinical sites, including ocular, mucocutaneous, respiratory and genital material, urines and necropsy tissue, were compared by enzyme immunoassay (EIA) and conventional culture for the presence of HSV. Results: The EIA performed to a high level of sensitivity and specificity using a variety of specimen types. Some problems were encountered using cervical swabs from pregnant women and necropsy brain tissue. Analysis of clinical and contact history data of most patients giving discrepant results supported the evidence of recent $\mathrm{HSV}$ infection obtained by EIA. The mean culture time was $2 \cdot 4$ days (range one to eight days).

Conclusions: The HSV EIA test performed to a high level of sensitivity $(93.7 \%)$ and specificity $(96.6 \%)$ when compared with culture using a variety of clinical material. These results assumed cell culture was $100 \%$ sensitive and specific. The actual performance of the EIA test is probably much higher. This approach to rapid HSV diagnosis should be used more widely, particularly in potentially serious cases.
\end{abstract}

Since the introduction of effective antiviral agents the rapid and accurate diagnosis of herpes simplex virus (HSV) infections has become imperative. Conventional serological techniques are rarely useful and may be difficult to interpret. Virus culture using susceptible cell monolayers, although easy and reliable, usually takes between two and five days.

Rapid detection techniques are important particularly in potentially serious situations, such as in immunocompromised patients, women presenting during late pregnancy, or in neonates. Direct HSV antigen detection using immunofluorescence is very specific but may have poor sensitivity and is very dependent on specimen quality. ${ }^{1}$ The use of enzyme immunoassay (EIA) for HSV antigen detection has been previously reported, both as a culture amplification technique ${ }^{23}$ and also as a direct detection method, ${ }^{45}$ as performing reliably, particularly in a genitourinary setting.
The availability of several EIA kits marketed for direct detection of HSV antigen should enable laboratories without tissue culture facilities to diagnose this infection quickly and accurately. Our aim was to establish the performance of one HSV EIA kit (IDEIA HSVNovo Nordisk Ltd) using specimens collected from any site which could conceivably be implicated in a potentially serious infection.

\section{Methods}

Nine hundred and twelve specimens comprising cerebrospinal fluids (CSF) $(n=115)$, eye swabs $(n=31)$, urines $(n=55)$, respiratory specimens $(n=67)$, cutaneous specimens $(n=84)$, other necropsy tissue $(n=16)$, necropsy brain tissue $(n=19)$ and genital swabs $(n=525)$ including 120 specimens from pregnant women.

Swabs were collected and placed directly into $2 \mathrm{ml}$ of virus transport medium (newborn calf serum $5 \%$ in Hanks's balanced salt solution). Samples of cerebrospinal fluid were diluted 1 in 1 with the medium and tissues were macerated in about $10 \% \mathrm{w} / \mathrm{v}$ in virus transport medium. This suspension was centrifuged at $2000 \times g$ for 10 minutes and the supernatant removed for testing. Urine was centrifuged at $2000 \times g$ for 10 minutes and the pellet was suspended in $2 \mathrm{ml}$ of virus transport medium.

Aliquots of each specimen were tested on cell culture as described below and also for herpes simplex antigen using the IDIEA HSV test (Novo Nordisk Ltd).

\section{CELL CULTURE}

About $200 \mu$ l of specimen was inoculated on to MRC5 or human amnion cell monolayers according to availability. The cell cultures were incubated for up to 14 days at $37^{\circ} \mathrm{C}$. Confirmation of the cytopathic effect by immunofluorescence (Imagen HSV1 and $2 \mathrm{kit}-$ Novo Nordisk Ltd) was performed if the cytopathic effect was atypical or if it appeared more than three days after inoculation. Confirmation by immunofluorescence was also performed when cytopathic effects occurred in EIA negative specimens and also on isolates from patients without overt characteristic mucocutaneous lesions.

\section{IDEIA HSV TEST}

Before testing, $40 \mu \mathrm{l}$ of IDEIA HSV spiking medium was added to $160 \mu \mathrm{l}$ of specimen in virus transport medium and left to stand for at least one minute. The enzyme immunoassay was performed exactly according to the manufacturers' instructions. 
Table 1 HSV detection results on 912 specimens obtained by comparing HSV IDEIA and culture

\begin{tabular}{llll}
\hline & \multicolumn{3}{c}{ Cell culture } \\
\cline { 3 - 4 } & & Positive & Negative \\
IDEIA \\
HSV test $\}$ & Positive & 134 & 26 \\
Negative & 9 & 743 \\
\hline
\end{tabular}

Percentage correlation: $\mathbf{9 6 \cdot 2 \%}$

Sensitivity: $93.7 \%$

Specificity: $96.6 \%$

PVP: $\mathbf{8 3 . 8 \%}$

PVN: $\mathbf{9 8} \cdot \mathbf{8} \%$

\section{Results}

The prevalence of HSV in this study was $15.7 \%$ by cell culture (tables 1 and 2 ). The overall sensitivity of the EIA was $93.7 \%$, specificity $96.6 \%$, positive and negative predictive values $83.8 \%$ and $98.8 \%$, respectively. The mean culture time was $2 \cdot 4$ days (range one to eight days).

The analysis of EIA compared with culture of different specimen types is shown in table 2 . The sensitivity and specificity of the EIA compared very well with culture using most specimen types, with the exception of postmortem brain tissue and specimens from pregnant women.

Nine specimens were EIA negative but culture positive presumably due to the presence of the low titre of virus which was amplified by culture.

Twenty six specimens were culture negative but positive by the IDEIA HSV test. With the exception of one, all of the remaining specimens were received within 24 hours of collection. Acyclovir had not been given to any of the patients at the time of specimen collection. Of the culture negative, EIA positive specimens, further information supports the diagnosis of $\mathrm{HSV}$ infection in 14 cases (17 specimens). Eight specimens were from patients who were positive for HSV at another site, had an infected partner, or were serologically positive (HSV IgM positive). Nine specimens were from patients with a history of HSV infection or who had been clinically diagnosed as having HSV infection. The reallocated data, if these 17 results are considered false negative cell culture results, are shown in table 3.

Necropsy brain tissue accounted for six of the remaining nine discrepant results. Brain tissue was obtained from 19 patients, 11 of whom had died of suspected viral encephalitis, although only two had had serological evidence of recent HSV infection. Eight had died of sudden non-infectious causes such as road traffic accidents or myocardial infarction. Interestingly, the six culture negative, EIA positive specimens were obtained from the former group and had optical densities (OD) of less than twice the cutoff value. Brain tissue from the two patients with serological evidence of recent HSV infection were HSV culture positive and gave high optical density readings by EIA $(>2 \cdot 0)$.

Of the 115 specimens of cerebrospinal fluid examined from adults and neonates, 114 were EIA negative and culture negative, and one was EIA positive and culture negative. The EIA reaction was successfully neutralised using a polyclonal HSV antiserum.

The urine specimens were obtained from 16 patients with suspected bacteriological urinary tract infection, 11 patients with documented localised or systemic HSV infection, or 28 patients with suspected congenital or other viral infection. Seven culture positive and EIA positive urine specimens were, with one exception, from the middle group of patients. The exception was a woman with suspected bacteriological urinary tract infection. The two specimens that were culture negative and EIA positive were from patients with $\mathrm{HSV}$ infection from whom virus had been isolated.

\section{Discussion}

Rapid, direct detection of HSV antigen in patients predisposed to potentially serious HSV infections-neonates or the immunocompromised-is clearly desirable.

The IDEIA HSV test performed to a high level of sensitivity $(93.7 \%)$ and specificity $(96.6 \%)$ using a wide variety of specimen types. Nine of 55 urine samples examined were EIA positive, seven confirmed by culture, including one from a patient with ocular symptoms only. The remaining six were from patients with either systemic HSV infection or from those with genital lesions. In the latter cases contamination during micturition cannot be discounted. Of the two EIA positive and culture negative urine samples, one was from a patient with confirmed HSV genital infection and the other from an infected neonate.

Five of 120 genital specimens obtained from antenatal patients were EIA positive and culture negative. Four specimens were from patients who had a history of HSV and successful HSV culture from another specimen taken

Table 2 Results of EIA compared with culture in different specimen groups

\begin{tabular}{|c|c|c|c|c|c|c|c|}
\hline $\begin{array}{l}\text { Specimen } \\
\text { group }\end{array}$ & $\begin{array}{l}\text { EIA + } \\
\text { culture + }\end{array}$ & $\begin{array}{l}\text { EIA + } \\
\text { culture - }\end{array}$ & $\begin{array}{l}\text { EIA - } \\
\text { culture + }\end{array}$ & $\begin{array}{l}\text { EIA - } \\
\text { culture - }\end{array}$ & Total & $\begin{array}{l}\text { Sensitivity of } \\
\text { EIA (\%) }\end{array}$ & $\begin{array}{l}\text { Specificity of } \\
E I A(\%)\end{array}$ \\
\hline $\begin{array}{l}\text { Cerebrospinal fluid } \\
\text { Eyes } \\
\text { Urine } \\
\text { Respiratory } \\
\text { Cutaneous } \\
\text { Necropsy tissue } \\
\text { Necropsy brain } \\
\text { Genital } \\
\text { (non-antenatal) }\end{array}$ & $\begin{array}{r}0 \\
7 \\
7 \\
20 \\
21 \\
6 \\
2 \\
68\end{array}$ & $\begin{array}{l}1(1) \\
1(1) \\
2(2) \\
1 \\
2(1) \\
0 \\
6 \\
8(8)\end{array}$ & $\begin{array}{l}\mathbf{0} \\
\mathbf{0} \\
\mathbf{0} \\
\mathbf{0} \\
\mathbf{1} \\
\mathbf{0} \\
\mathbf{0} \\
\mathbf{7}\end{array}$ & $\begin{array}{r}144 \\
23 \\
46 \\
46 \\
60 \\
10 \\
11 \\
322\end{array}$ & $\begin{array}{r}115 \\
31 \\
55 \\
67 \\
84 \\
16 \\
19 \\
405\end{array}$ & $\begin{array}{l}\text { Not calculated } \\
100 \\
100 \\
100 \\
95 \\
100 \\
100 \\
91\end{array}$ & $\begin{array}{r}99 \\
96 \\
96 \\
98 \\
97 \\
100 \\
65 \\
98\end{array}$ \\
\hline $\begin{array}{l}\text { Genital } \\
\text { (antenatal) }\end{array}$ & 3 & $5(4)$ & 1 & 111 & 120 & 75 & 96 \\
\hline
\end{tabular}

Results in ( ) indicate false negative culture results as supported by other information. 
Table 3 Results after reallocation of 17 EIA positive and culture negative specimens

$\begin{array}{llll}\hline & & \multicolumn{3}{c}{\text { Cell culture }} \\
$\cline { 3 - 4 } & & \text {$\left.Positive } & \text { Negative } \\
\text { IDEIA } \\
\text { HSV test }\end{array}\right\}$\begin{tabular}{lll} 
Positive & 151 & 9 \\
Negative & 9 & 743 \\
\hline
\end{tabular}

Percentage correlation: $\mathbf{9 8 . 0 \%}$

Sensitivity: $94 \cdot 4 \%$

Specificity: $\mathbf{9 8 . 8 \%}$

PVP: $94.4 \%$

PVN: $\mathbf{9 8 \cdot 8} \%$

during the same episode. Relevant information was unavailable for the remaining specimen. These results should be interpreted cautiously in the absence of data from a control group of pregnant women with no history of HSV infection.

Examination of the cerebrospinal fluid for HSV antigen is not a new concept. ${ }^{6}$ Of the 115 such samples examined in this study, only one was EIA positive but culture negative, although specifically neutralised using a polyclonal HSV antiserum. This patient subsequently died from disseminated $\mathrm{HSV}$ infection. No other supporting evidence for HSV encephalitis was available from the remaining 114 patients. Specimens were taken, in most cases within four days of onset of neurological symptoms, but an earlier study ${ }^{6}$ showed that $\mathrm{HSV}$ antigen is more likely to be detected in cerebrospinal fluid taken more than 10 days after onset of symptoms. A recent report ${ }^{7}$ indicates that HSV genome fragments were detected in the cerebrospinal fluid by the polymerase chain reaction (PCR) in 41 of 43 patients with herpes encephalitis at the time of hospital admission. The advantage of EIA over the PCR in allowing diagnostic laboratories to perform rapid HSV detection, is undeniable, but the sensitivity of the former assay when testing the cerebrospinal fluid must remain questionable until more positive specimens have been tested. Specificity problems were not encountered when using cerebrospinal fluid samples in the present study.

Necropsy brain tissue was obtained from two groups of patients - one group who had died of suspected viral encephalitis $(n=11)$ and one group of patients who had died suddenly of a non-infectious cause, such as a road traffic accident or myocardial infarction $(n=8)$. Serum samples from all patients were tested for HSV antibody by immunofluorescence. Concordant negative antigen detection and culture results were found in the latter group of patients where stress was likely to have been short-lived or absent at the time of death. Seven of these patients had a history of HSV infection by serology. Brain tissue from eight of 11 patients in the former group, however, was EIA positive. Two results were confirmed by culture. The serological picture in these two patients was consistent with recent HSV infection or reactivation. The remaining six EIA positive and culture negative brain specimens had an OD of less than twice the cutoff value (compared with an OD of $>2.0$ in the two brain samples with confirmed HSV infection) and no supporting serological criteria of recent $\mathrm{HSV}$ infection, although there was evidence of previous infection. The stress of a severe infection caused by another agent might have reactivated low level or non-viable HSV antigen. Until this issue is resolved EIA results obtained using brain tissue should be interpreted cautiously in the absence of other supporting evidence. This phenomenon was not observed when using other necropsy tissue.

In conclusion, the IDEIA HSV test performed to a high level of sensitivity $(93.7 \%)$ and specificity (96.6\%) when compared with culture using a wide variety of clinical material. These results assumed that cell culture was $100 \%$ sensitive and specific. Examination of clinical and contact history data of most patients giving discrepant results, however, supported evidence of recent HSV infection. The actual performance of the HSV IDEIA test is probably much higher than the figures calculated on the unadjusted data would suggest. The assay is simple, reproducible, and rapid, with the advantages over culture in speed (four hours compared with $2 \cdot 4$ days) and problems caused by bacterial contamination of specimens and when examining material from patients with healing lesions or who have received antiviral treatment. This approach to HSV diagnosis should be used more widely, particularly in potentially serious situations.

I am grateful to Miss Claire Stuart for typing the manuscript and to Novo Nordisk Diagnostics Ltd for supplying the HSV IDEIA kits.

1 Volpi A, Lakeman A, Pereira L, Stagno S. Monoclonal antibodies for rapid diagnosis and typing of genital herpes infections in pregnancy. Am J Obstet Gynecol 1983; 146:813-5.

2 Datson D, Carter N. Assessment of new enzyme immunoassay to detect herpes simplex antigen. Genitourin Med 1988;64:65.

3 Warford AL, Levy RA, Rekrut KA. Evaluation of a commercial enzyme-linked immunosorbent assay for detection of herpes simplex virus antigen. J Clin Microbiol 1984;20:490-3.

4 Rodgers T, Foster JR. Clinical evaluations of the IDEIA ${ }^{\text {TM }}$ HSV Test. In: Freke A, Sherwood D, eds. Herpes simplex virus: new technologies in diagnostics. Nottingham, Boots Celltech Diagnostics, 1987.

5 Skar AG, Middledorp J, Gundersen T, Rollag H, Degre M. Rapid diagnosis of genital herpes simplex infection by an indirect ELISA method. NIPH Ann 1988;2:59-65.

6 Coleman RM, Bailey PD, Whitley RJ, Keyserling H, Nahmias AJ. Elisa for the detection of herpes simplex Nahmias AJ. Elisa for the detection of herpes simplex
virus antigens in the cerebrospinal fluid of patients'with virus antigens in the cerebrospinal fluid of pati
encephalitis. $J$ Virol Methods 1983;7:117-25.

7 Aurelius E, Johansson B, Skoldenberg B, Statland A, Forsgren M. Rapid diagnosis of herpes simplex encephalitis by nested polymerase chain reaction assay of cerebrospinal fluid. Lancet 1991;337:189-92. 